\title{
Análisis del impacto de los Fondos Europeos en Andalucía: 2000-2006*
}

\author{
MANUEL ALEJANDRO CARDENETE FLORES \\ European Commission (JRC-IPTS), Departamento de Economía, Métodos Cuantitativos e \\ Historia Económica, UNIVERSIDAD PABLO DE OLAVIDE, ESPAÑA. E-mail: \\ macardenete@upo.es
}

\section{MARÍA DEL CARMEN DELGADO LÓPEZ}

Departamento de Economía, Métodos Cuantitativos e Historia Económica, UNIVERSIDAD PABLO DE OLAVIDE, ESPAÑA. E-mail: mcdellop@upo.es

\section{PATRICIA D. FUENTES SAGUAR}

Departamento de Economía, Métodos Cuantitativos e Historia Económica, UNIVERSIDAD PABLO DE OLAVIDE, ESPAÑA. E-mail: pfuesag@upo.es

\section{RESUMEN}

Después de dos décadas en las que Andalucía ha sido objeto de la financiación de los Fondos Estructurales Europeos, con el objetivo de desarrollar a través de estas inversiones sectores que impulsan el crecimiento económico en la región, este trabajo ${ }^{1}$, propone un análisis que permitirá conocer el impacto económico de los Fondos Europeos recibidos por la Comunidad Autónoma de Andalucía durante el período 2000-2006. Para ello, se utilizará la matriz de contabilidad social regional del año 2005 y un modelo de multiplicadores lineales. Los resultados subrayan una contribución significativa de los Fondos Europeos para el crecimiento de la región para el período analizado (20002006).

Palabras clave: Matriz de Contabilidad Social, Modelo de Multiplicadores Lineales, Política Regional Europea, Análisis de Impacto.

\section{Impact Assessment of the European Funds in Andalusia: 2000- 2006}

\begin{abstract}
After two decades in which Andalusia has been receiving financing from the European Structural Funds with the objective to develop through these investments the key sectors that boost economic growth in the region, this paper proposes an analysis that will reveal the economic impact of the European Funds received by the Autonomous Community of Andalusia during the period 2000-2006. For this will use the Social Accounting Matrix of year 2005 and a model of linear multipliers. The results will underscore a significant contribution of the European Funds to the growth of the region for the period analyzed (2000-2006).

Keywords: Social Accounting Matrix, Multipliers Model, European Regional Policy, Impact Analysis.

Clasificación JEL: C67

\footnotetext{
* El primer autor agradece la financiación recibida de los proyectos MICINN-ECO2009-11857 y SGR2009-578l y SEJ479, el segundo autor al Proyecto 092-2011 del Centro de Estudios Andaluces. La opinión, análisis y resultados son responsabilidad de los autores y no implican la posición de la Comisión Europea.

${ }^{1}$ Agradecemos a la Prof. Dr. Carmen Lima por los datos facilitados sobre el reparto de Fondos por sectores.
}

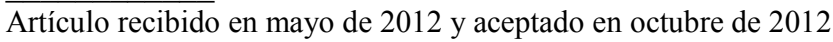

Artículo disponible en versión electrónica en la página www.revista-eea.net, ref. ə-31107 


\section{INTRODUCCIÓN}

Desde la adhesión de España a la Unión Europea, Andalucía ha sido una región receptora de Fondos Europeos. Catalogada como región Objetivo 1, supuso, desde el inicio, su inclusión entre las regiones beneficiarias de las acciones de la política regional programada por la Unión Europea, justificada por sus debilidades de tipo estructural, marcadas por los déficits de dotación de infraestructuras básicas.

Según la Dirección General de Fondos Comunitarios, el Objetivo 1 de los Fondos Estructurales constituye la principal prioridad de la política de cohesión de la Unión Europea. A ello obedece el hecho de que dos tercios de los créditos de los Fondos Estructurales (es decir, más de 135.000 millones de euros) se destinen a la recuperación de las regiones más desfavorecidas, las denominadas "regiones del Objetivo 1", con un Producto Interior Bruto (PIB) inferior al 75\% de la media comunitaria.

El proceso de convergencia de la economía andaluza hacia las regiones comunitarias en términos de PIB per cápita durante principios de los años 90 no fue el previsto, debido a la coyuntura económica por la que atravesaba la región. Fue a partir del año 1994 cuando experimentó un comportamiento positivo hacia la convergencia real con los estándares comunitarios en términos de PIB por habitante.

Para la evaluación del impacto que las políticas estructurales procedentes de la Unión Europea puedan tener en las regiones a las que van destinadas, se han realizado diversos trabajos macroeconómicos, empleando distintas metodologías. Entre estos modelos, los más comunes a la hora de evaluar la política regional en España son:

- El modelo HERMIN ${ }^{2}$, que tiene en cuenta tanto los efectos de la demanda como los de la oferta, especialmente los que atienden a cambios en la productividad y en la competitividad.

- El modelo QUEST II, basado en un modelo de crecimiento de carácter anticipativo.

- El modelo MOISEES creado por un grupo de economistas del Ministerio de Economía y Finanzas y del mundo académico asociados a la Secretaría de Estado de Presupuestos y Gastos del Ministerio de Hacienda. Este modelo, analiza principalmente el impacto de los Fondos en la demanda,

\footnotetext{
${ }^{2}$ Entre los trabajos que han aplicado esta metodología podemos encontrar: De la Fuente (2005) y Sosvilla (2007) para España, Sosvilla y Herce (2003) y Sosvilla y García (2006) para Madrid, Sosvilla (2003) para Canarias, Sosvilla, Bajo y Díaz (2003) para Castilla La Mancha, Sosvilla y Murillo (2005) para Andalucía.
} 
aunque los efectos de oferta de una mayor capacidad de producción debida a un aumento en el stock de capital también son tenidos en cuenta.

Los modelos de equilibrio general, que explican la realidad a través de hipótesis, y permiten calcular impactos de diferentes políticas económicas y realizar predicciones. Incluimos en este grupo, los modelos input - output, los modelos SAM lineales y los Modelos de Equilibrio General Aplicado. Entre los trabajos que han aplicado estas metodologías podemos encontrar: Dones y Pérez (2002) para España, Morillas, Moniche y Marcos (1999 y 2004), Murillo (2005 y 2007), Lima y Cardenete $(2005,2008)$ y Lima, Cardenete y Usabiaga (2010) para Andalucía, , Cámara y Marcos (2009) y Monrobel, Cámara y Marcos (2012) para la Comunidad de Madrid, Márquez, Ramajo y De Miguel (2010) para Extremadura y López, Monsalve y Zafrilla (2011) para Castilla-La Mancha. También podríamos incluir aquí, estudios como los de Cardenete (2004), Llano (2004), Cardenete y Llop (2005) y Llop (2007) entre otros, que utilizan las metodologías aquí descritas para diversos tipos de análisis.

En este trabajo se presenta un análisis encuadrado en los Modelos lineales de Equilibrio General, concretamente en los modelos SAM con los que se empleará un modelo de multiplicadores lineales, que permitirá conocer el impacto económico que tienen los Fondos Europeos recibidos por la Comunidad Autónoma de Andalucía durante el periodo 2000-2006. Este modelo es una extensión del modelo de Leontief, que siguiendo la misma metodología que estos modelos Input-Output, se basan en las propias identidades contables que se dan en la matriz y que permitirán relacionar inyecciones exógenas de renta con las rentas de las cuentas consideradas endógenas. De esta forma, podemos analizar el impacto de la ayuda comunitaria sobre todos los sectores de la economía andaluza, utilizando para ello como base de datos la matriz de contabilidad social de Andalucía para el año 2005.

\section{LOS FONDOS EUROPEOS DE ANDALUCÍA}

Andalucía se sitúa en el sur de Europa y de España con una superficie de $87.268 \mathrm{~km}^{2}$ y una población de 8.424 .102 habitantes. Es la comunidad autónoma más poblada de España y la segunda más extensa, lo que explica su peso en el conjunto de España.

Desde el punto de vista sectorial, en Andalucía tiene un fuerte peso el sector servicios, que ha ido evolucionando de forma que la experiencia acumulada durante las tres últimas décadas y el ritmo sostenido de crecimiento que ha presentado durante todo este tiempo explican su consolidación y expansión; el sector primario conserva un peso muy significativo, constituyendo el sector agroalimentario un componente básico de la economía andaluza, tanto por el peso y posición que ocupa en su estructura económica como por su importancia decisiva en las exportaciones. Por último el sector industrial presenta un peso 
relativamente reducido con relación al conjunto de España y la Unión Europea, pero la participación de la construcción es elevada y genera que el conjunto del sector secundario no sea sustancialmente diferente del de su entorno.

A lo largo de dos décadas de percepción de ayudas procedentes de la Unión Europea, cada periodo de planificación plurianual ha ido destinado a financiar distintas medidas. Así, en el periodo 1989-93 se financiaron mayoritariamente infraestructuras de tipo físico orientadas a la promoción de la actividad económica, mientras que en el periodo 1994-99 se mantuvieron las inversiones en infraestructuras además de promover la capacitación del capital humano.

Por otro lado, en el periodo 2000-06, la justificación de la necesidad de una fuerte intervención cofinanciada a través de la política regional comunitaria según el Plan de Desarrollo Regional para Andalucía 2000-2006, se basaba en combatir las debilidades estructurales que caracterizaban a la economía andaluza, entre las que encontramos, una insuficiente articulación interna que dificultaba las relaciones intersectoriales, una baja cualificación del capital humano y una debilidad del sector exterior poco diversificado y alejado de la ramas de alto valor añadido.

Por esto, en el Marco de Apoyo Comunitario (MAC) 2000-2006, la financiación para las zonas Objetivo 1 refleja las prioridades establecidas para asentar los factores de desarrollo sostenible de la región. Dicha financiación irá destinada a la conclusión de redes de transporte y energía (Eje 6), a la preservación del medio ambiente, el entorno natural y los recursos hídricos (Eje 3), la mejora de la competitividad y el desarrollo del tejido productivo, apoyando la innovación y la modernización empresarial (Ejes 1,2,7), la potenciación de los recursos humanos (Ejes 4, 4B, 4C, 4D, 4E) y por último la cohesión social y territorial (Eje 5).

Con este destino de los Fondos, Andalucía ha recibido durante el septenio 2000-2006 11.708,9 millones de euros. De esta cifra 7.840,4 millones de euros son la ayuda subvencionable financiada por la Comisión Europea (67\%) y el resto 3.868,5 millones de euros son aportación española (33\%).

\section{FONDOS EUROPEOS RECIBIDOS EN ANDALUCÍA 2000- 2006}

A pesar de la concentración de ayudas comunitarias en la región, los datos mostraban que la convergencia andaluza hacia los valores de las regiones europeas parecía ser más lenta que la nacional. Según datos del Sexto Informe periódico sobre la situación socioeconómica de la región, publicado por la Comisión Europea en 1986, el PIB per cápita de Andalucía era del 52,8\% de la media de la UE-15 pasando al 57,2\% en 1996. Por este motivo, el 28 de abril de 2000, el Gobierno de España presentó a la Comisión Europea tres proyectos de programas operativos monofondo para Andalucía, uno para cada Fondo Es- 
tructural (FEDER, FEOGA-Orientación y FSE). Tras las negociaciones con la Comisión, el 15 de septiembre de 2000 se presentó una versión que comprendía las tres anteriores, pasando a ser un programa integrado, que fue aprobado el 29 de diciembre de 2000 como Programa Operativo Integrado de Andalucía (POIA), con un desglose muy exhaustivo en el que se detallaban las más de 70 medidas diferentes con la descripción correspondiente, que desarrollan los 9 ejes prioritarios de actuación con la siguiente distribución indicativa de participación comunitaria: FEDER 6.152,7 millones de euros (78,5\%), FEOGA-O 755,2 millones de euros (9,5\%) y FSE 932,5 millones de euros (12\%).

A continuación se presenta la distribución el gasto total programado para el periodo objeto de estudio.

Tabla 1

Plan Financiero POIA 2000-2006, detalle por eje prioritario (miles de euros)

\begin{tabular}{|l|c|}
\hline \multicolumn{1}{|c|}{ Ejes } & Gasto Programado \\
\hline 1. Mejora de la competitividad y desarrollo del tejido productivo & 1.225 .022 \\
\hline 2. Sociedad del conocimiento (innovación, I+D, sociedad de la información) & 342.441 \\
\hline 3. Medio ambiente, entono natural y recursos hídricos & 2.996 .057 \\
\hline 4.1. Infraestructura educativa y refuerzo de la educación técnico-profesional & 757.646 \\
\hline 4.2. Inserción y reinserción ocupacional de los desempleados & 256.166 \\
\hline 4.3. Refuerzo de la estabilidad en el empleo y adaptabilidad & 154.754 \\
\hline 4.4. Integración en el mercado de trabajo de las personas con especiales dificultades & 98.133 \\
\hline 4.5. Participación de las mujeres en el mercado de trabajo & 116.145 \\
\hline 5. Desarrollo local y urbano & 525.696 \\
\hline 6. Redes de transporte y energía & 4.749 .079 \\
\hline 7. Agricultura y desarrollo rural & 434.333 \\
\hline 9. Asistencia técnica & 53.428 \\
\hline TOTAL & 11.708 .900 \\
\hline
\end{tabular}

Fuente: Elaboración propia a partir del POIA 2000-2006.

El programa es un conjunto de actuaciones de responsabilidad de diferentes organismos de la Administración General del Estado y de la Junta de Andalucía. El desglose por fuentes de financiación revela que el 49,8\% de los Fondos, es decir, 3.910,8 millones de euros son gestionados por la Comunidad Autónoma y el restante 50,2\%, que asciende a 3.929,6 millones de euros por la Administración General del Estado.

Cabe señalar, que en este trabajo nos vamos a centrar en el estudio de los Fondos Estructurales recibidos por dos razones: una porque concentran la mayor parte de las ayudas y otra porque se gestionan y desarrollan desde el ámbito 
regional. En la evaluación del impacto de los Fondos se consideran además de la ayuda subvencionable comunitaria la cofinanciación obligatoria nacional.

Siguiendo a Cámara y Marcos (2009), para realizar este análisis se establecerán dos escenarios alternativos:

- El escenario base o de referencia, que surge al eliminar en la base de datos todos los Fondos Estructurales recibidos en el año 2005, para partir de una situación sin Fondos.

- Un segundo escenario en el que la economía de Andalucía sin Fondos se le inyectan todos los Fondos Estructurales recibidos en el año 2005, para así realizar un análisis de la aportación de cada Fondo.

\section{LOS MODELOS DE MULTIPLICADORES LINEALES}

Este estudio aplica la metodología de los modelos de multiplicadores lineales para el estudio del impacto de los Fondos Estructurales sobre Andalucía. La Teoría de multiplicadores fue iniciada por Stone (1962), y Pyatt y Round (1979), desarrollándose posteriormente con trabajos como los de Defourney y Thorbecke (1984). Estos métodos se basan en obtener información, a partir de las matrices inversas derivadas de los modelos de Leontief (1941) y de Ghosh (1958) aplicados a la MCS, sobre la capacidad que tiene un sector de expandir aumentos de demanda o de costes, respectivamente.

Siguiendo a Cardenete, Fuentes y Polo (2010), comenzamos con una breve explicación de estos modelos, como extensión del modelo de Leontief, de la siguiente forma: se considera una matriz cuadrada $n x n$ donde cada fila y cada columna representan una cuenta económica (sectores productivos, consumidores, gobierno, cuenta de capital, etc.) que satisface las igualdades contables de la economía (total renta igual a total gasto). Cada componente $Y_{i j}$ de la matriz representa el flujo bilateral entre la cuenta $i$ y la cuenta $j$. Cada fila de la MCS recoge el total de ingresos que la fila $i$ recibe de las columnas $j$; las columnas muestran el total de renta de la columna $j$ y como se distribuye entre las distintas cuentas $i$. Los coeficientes medios de gasto: $a_{i j}=Y_{i j} / Y_{j}, i, j=1, \ldots, n$., muestran los pagos a la cuenta $i$ por unidad de renta de $j$. De esta definición se puede obtener:

$$
Y_{i}=\sum_{j=1}^{n}\left(Y_{i j} / Y_{j}\right) Y_{j}=\sum_{j=1}^{m} a_{i j} Y_{j}+\sum_{j=m+1}^{m+k} a_{i j} Y_{j} ; n=m+k .
$$

Los índices $m$ y $k$ representan la división de las cuentas de la MCS entre endógenas y exógenas, lo que divide la matriz nxn en 4 submatrices: $A_{m m}, A_{m k}, A_{k m}$, y $A_{k k}$. $Y_{m}$ e $Y_{k}$ denotan la renta total de las cuentas endógenas y exógenas respectivamente, por lo que se puede despejar $Y_{m}$ y obtener $Y_{m}=A_{m m} Y_{m}+A_{m k} Y_{k}$, y desde ahí, siguiendo el mismo procedimiento que con la ecuación de Leontief, 
obtener la matriz de multiplicadores extendidos a partir de $Y_{m}=\left(I-A_{m m}\right)^{-1} Z$, siendo $Z$ el vector de las columnas exógenas ${ }^{3}\left(A_{m k} Y_{k}\right)$, y $M=\left(I-A_{m m}\right)^{-1}$ la matriz de los multiplicadores extendidos de la MCS, que pueden interpretarse como las necesidades de inputs por incrementos unitarios de gasto o renta (según hablemos de columnas o filas) en una cuenta, de manera similar a la que tiene la conocida como inversa de Leontief, con la diferencia de que sí que capta las relaciones entre la producción, la renta de los factores, la distribución de la renta y la demanda final. Hay que señalar que la selección de $m$ (es decir, la decisión de qué cuentas son endógenas) suele depender del análisis que se vaya a realizar, y en función de eso se decide qué cuentas son las que explican (exógenas) cambios en la renta de otras cuentas (endógenas). Si llamamos $d Z$ a los cambios en el vector de cuentas exógenas, los cambios en la renta de las cuentas endógenas serían ${ }^{4}$ :

$$
d Y_{m}=M d Z=M d\left(A_{m k} Y_{k}\right)=M A_{m k} d Y_{k}
$$

La columna $i$-ésima de $M$ indica las rentas totales generadas en cada una de las cuentas endógenas cuando se produce un flujo unitario de renta desde las instituciones exógenas hacia la cuenta endógena $i$. En la simulación que se realizará en este trabajo, el nuevo vector se obtiene restando al vector $Z$ todas las inyecciones de renta que reciben las ramas de actividad procedentes de los Fondos Estructurales.

A continuación se clasifican las cuentas de la matriz de contabilidad social SAMAND $05^{5}$ en endógenas y exógenas, quedando un total de 29 cuentas endógenas y 7 cuentas exógenas:

Tabla 2

División de las cuentas de la SAMAND05 en endógenas y exógenas

\begin{tabular}{|l|l|}
\cline { 2 - 2 } \multicolumn{1}{c|}{} & Cuentas Endógenas \\
\hline 1 & Agricultura \\
\hline 2 & Ganadería \\
\hline 3 & Pesca \\
\hline 4 & Extracción de productos energéticos \\
\hline 5 & Resto de extractivas \\
\hline 6 & Refino de petróleo y tratamiento de residuos nucleares \\
\hline 7 & Producción y distribución de energía eléctrica \\
\hline 8 & Producción y distribución de gas, vapor de agua y agua caliente \\
\hline
\end{tabular}

\footnotetext{
${ }^{3}$ La submatriz $A_{m k}$ representa cómo se reparten los flujos de renta de las cuentas exógenas entre las cuentas endógenas.

${ }^{4}$ Polo, Roland-Host, y Sancho (1990).

${ }^{5}$ Fuentes (2012).
} 
Tabla 2 (continuación)

División de las cuentas de la SAMAND05 en endógenas y exógenas

\begin{tabular}{|c|c|}
\hline & Cuentas Endógenas (continuación) \\
\hline 9 & Captación, depuración y distribución de agua \\
\hline 10 & Alimentación \\
\hline 11 & Textil y piel \\
\hline 12 & Elaborados de madera \\
\hline 13 & Químicas \\
\hline 14 & Minería y siderurgia \\
\hline 15 & Elaborados metálicos \\
\hline 16 & Maquinaria \\
\hline 17 & Vehículos \\
\hline 18 & Materiales de construcción \\
\hline 19 & Transporte \\
\hline 20 & Otras manufacturas \\
\hline 21 & Construcción \\
\hline 22 & Resto de comercio \\
\hline 23 & Transporte y comunicaciones \\
\hline 24 & Otros servicios \\
\hline 25 & Servicios destinados a la venta \\
\hline 26 & Servicios no destinados a la venta \\
\hline 27 & Trabajo \\
\hline 28 & Capital \\
\hline \multirow[t]{2}{*}{29} & Consumo \\
\hline & Cuentas Exógenas \\
\hline 30 & FBK \\
\hline 31 & Cotizaciones sociales empleadores \\
\hline 32 & Impuestos indirectos Netos \\
\hline 33 & Cotizaciones sociales empleados \\
\hline 34 & IRPF \\
\hline 35 & Sector Público \\
\hline 36 & Sector Exterior (importaciones) (CIF) \\
\hline
\end{tabular}

Fuente: Elaboración propia a partir de Fuentes (2012).

\section{ESCENARIO BASE: LA ECONOMÍA DE ANDALUCÍA SIN FONDOS ESTRUCTURALES}

Para partir de un escenario sin Fondos, debemos detraer todos los Fondos recibidos durante el año 2005. Esto se realizará suprimiendo en la matriz de contabilidad social SAMAND05 todas las inyecciones de rentas recibidas en ese año. Una vez suprimidos todos los Fondos Estructurales recibidos por Andalucía, se procederá al desglose de éstos, estudiando uno a uno los impactos de cada Fondo sobre la economía. 
Las cantidades correspondientes a cada Fondo Estructural, que se extraerán de la economía son:

Tabla 3

Distribución financiera de la aportación comunitaria y nacional 2005

(miles de euros)

\begin{tabular}{|c|c|}
\hline Fondos Estructurales & Recursos \\
\hline FEDER & 863.438 \\
\hline FEOGA-O & 106.008 \\
\hline FSE & 130.830 \\
\hline COFINANCIACIÓN NACIONAL & 528.419 \\
\hline TOTAL & $\mathbf{1 . 6 2 8 . 6 9 5}$ \\
\hline
\end{tabular}

Fuente: Elaboración propia a partir del POIA 2000-2006.

Para establecer una regla de reparto de los recursos procedentes de los Fondos Estructurales entre las distintas ramas de actividad sobre las que recaen las inversiones, se ha replicado la regla de reparto del trabajo de Lima, Cardenete y Usabiaga (2010), en la que para poder trabajar con la información sobre los Fondos desagregada en función de los sectores productivos de la SAMAND05, se construye una matriz de reparto (R). Dicha matriz permite traducir las dotaciones asignadas a cada eje prioritario de actuación en las correspondientes cuentas de la SAMAND05. $\mathrm{R}_{12 \times 26}$ es una matriz con un número de filas igual al número de ejes y subejes (12) y un número de columnas equivalente a los sectores productivos de la SAMAND05 (26). A continuación, se define un vector de Fondos $\mathrm{F}_{1 \times 12}$ que contiene los datos de los Fondos que deseamos analizar y que extraemos de la información disponible y reflejada en la Tabla 1. De esta forma, la matriz $\mathrm{R}$ y el vector $\mathrm{F}$ nos permitirán calcular el vector de Fondos sectoriales $\left(\mathrm{FS}_{1 \times 26}\right)$ que representará la cuantía total de los Fondos recibidos anualmente teniendo en cuenta la inflación.

$$
\mathrm{FS}=\mathrm{FR}
$$

A continuación, en la Tabla 4, se muestra por columnas el vector FS utilizado para realizar las simulaciones, en el que se puede observar como la cuantía total de los Fondos recibidos en el año 2005 ha sido desagregada entre las cuentas de la SAMAND05, una vez aplicada la regla de reparto. 
Tabla 4

Asignación de los Fondos Estructurales 2005 (FEDER, FEOGA-O y FSE)

a las ramas de actividad

(miles de euros)

\begin{tabular}{|c|c|c|}
\hline & Ramas de Actividad & Fondos Estructurales \\
\hline 1 & Agricultura & 67.886 \\
\hline 2 & Ganadería & 86.264 \\
\hline 3 & Pesca & 26.525 \\
\hline 4 & Extracción de productos energéticos & 4.104 \\
\hline 5 & Resto de extractivas & 4.337 \\
\hline 6 & Refino de petróleo y tratamiento de residuos nucleares & 22.910 \\
\hline 7 & Producción y distribución de energía eléctrica & 8.760 \\
\hline 8 & Producción y distribución de gas, vapor de agua y agua caliente & 8.635 \\
\hline 9 & Captación, depuración y distribución de agua & 1.206 \\
\hline 10 & Alimentación & 36.580 \\
\hline 11 & Textil y piel & 9.963 \\
\hline 12 & Elaborados de madera & 8.953 \\
\hline 13 & Químicas & 18.510 \\
\hline 14 & Minería y siderurgia & 13.101 \\
\hline 15 & Elaborados metálicos & 11.430 \\
\hline 16 & Maquinaria & 162.652 \\
\hline 17 & Vehículos & 16.139 \\
\hline 18 & Materiales de construcción & 9.547 \\
\hline 19 & Transporte & 11.007 \\
\hline 20 & Otras manufacturas & 16.599 \\
\hline 21 & Construcción & 576.619 \\
\hline 22 & Resto de comercio & 80.699 \\
\hline 23 & Transporte y comunicaciones & 15.950 \\
\hline 24 & Otros servicios & 92.416 \\
\hline 25 & Servicios destinados a la venta & 110.875 \\
\hline \multirow[t]{2}{*}{26} & Servicios no destinados a la venta & 207.028 \\
\hline & Total & 1.628 .695 \\
\hline
\end{tabular}

Fuente: Elaboración propia a partir de Lima, Cardenete y Usabiaga (2010).

\section{ESTIMACIÓN DEL IMPACTO DE LOS FONDOS ESTRUCTURALES SOBRE LA ECONOMÍA DE ANDALUCÍA EN EL AÑO 2005}

Para obtener el impacto de los Fondos Estructurales de la economía andaluza, se va a generar una disminución de los ingresos procedentes de las cuentas exógenas eliminando los ingresos obtenidos a través de la ayuda comunitaria y la cofinanciación nacional. 
Una vez recogida la regla de reparto y la asignación de los Fondos Estructurales en el epígrafe anterior, pasamos ahora a mostrar los resultados obtenidos. En la tabla expuesta a continuación, se muestra la variación de los vectores $\mathrm{Z}$ e $\mathrm{Y}$ al suprimir todas las cantidades que reciben las ramas de actividad durante el año 2005 en los que se incluyen Fondos FEDER, FEOGA-O y FSE.

Tabla 5

Variación del output total al suprimir los Fondos Estructurales del año 2005 (miles de euros)

\begin{tabular}{|c|c|c|c|c|c|c|}
\hline & Sectores Productivos & $\mathbf{z}$ & ZsinFONDOS & $\mathbf{Y}$ & YsinFONDOS & $\begin{array}{c}\text { Variación } \\
(\%)\end{array}$ \\
\hline 1 & Agricultura & 3.585 .400 & 3.517 .514 & 9.451 .946 & 9.317 .521 & $-1,42$ \\
\hline 2 & Ganadería & 578.466 & 492.202 & 2.508 .174 & 2.397 .902 & $-4,40$ \\
\hline 3 & Pesca & 215.504 & 188.979 & 841.079 & 806.957 & $-4,06$ \\
\hline 4 & $\begin{array}{l}\text { Extracción de productos } \\
\text { energéticos }\end{array}$ & 10.638 & 6.534 & 6.341 .186 & 6.270 .393 & $-1,12$ \\
\hline 5 & Resto de extractivas & 91.789 & 87.452 & 2.438 .198 & 2.397 .830 & $-1,66$ \\
\hline 6 & $\begin{array}{l}\text { Refino de petróleo y tratamiento } \\
\text { de residuos nucleares }\end{array}$ & 5.936 .003 & 5.913 .093 & 13.795 .932 & 13.675 .231 & $-0,87$ \\
\hline 7 & $\begin{array}{l}\text { Producción y distribución de } \\
\text { energía eléctrica }\end{array}$ & 52.767 & 44.007 & 3.374 .895 & 3.321 .897 & $-1,57$ \\
\hline 8 & $\begin{array}{l}\text { Producción y distribución de gas, } \\
\text { vapor de agua y agua caliente }\end{array}$ & 0 & -8.635 & 1.152 .364 & 1.127 .096 & $-2,19$ \\
\hline 9 & $\begin{array}{l}\text { Captación, depuración y } \\
\text { distribución de agua }\end{array}$ & 11.157 & 9.951 & 1.077 .878 & 1.061 .905 & $-1,48$ \\
\hline 10 & Alimentación & 6.848 .087 & 6.811 .507 & 22.059 .432 & 21.835 .842 & $-1,01$ \\
\hline 11 & Textil y piel & 642.907 & 632.944 & 4.057 .317 & 4.006 .823 & $-1,24$ \\
\hline 12 & Elaborados de madera & 508.135 & 499.182 & 3.633 .031 & 3.580 .446 & $-1,45$ \\
\hline 13 & Químicas & 3.328 .134 & 3.309 .624 & 9.717 .951 & 9.619 .169 & $-1,02$ \\
\hline 14 & Minería y siderurgia & 2.403 .879 & 2.390 .778 & 6.120 .579 & 6.051 .664 & $-1,13$ \\
\hline 15 & Elaborados metálicos & 1.063 .907 & 1.052 .477 & 4.491 .778 & 4.419 .069 & $-1,62$ \\
\hline 16 & Maquinaria & 3.822 .454 & 3.659 .802 & 12.419 .051 & 12.117 .231 & $-2,43$ \\
\hline 17 & Vehículos & 2.808 .002 & 2.791 .863 & 6.841 .328 & 6.776 .931 & $-0,94$ \\
\hline 18 & Materiales de construcción & 732.052 & 722.505 & 6.601 .851 & 6.479 .280 & $-1,86$ \\
\hline 19 & Transporte & 958.040 & 947.033 & 1.842 .091 & 1.819 .241 & $-1,24$ \\
\hline 20 & Otras manufacturas & 2.145 .350 & 2.128 .751 & 7.131 .772 & 7.052 .847 & $-1,11$ \\
\hline 21 & Construcción & 27.372 .419 & 26.795 .800 & 46.039 .271 & 45.109 .582 & $-2,02$ \\
\hline 22 & Resto de comercio & 2.114 .838 & 2.034 .139 & 38.401 .833 & 37.888 .541 & $-1,34$ \\
\hline 23 & Transporte y comunicaciones & 1.907 .366 & 1.891 .416 & 18.400 .081 & 18.162 .850 & $-1,29$ \\
\hline 24 & Otros servicios & 16.518 .030 & 16.425 .614 & 41.327 .978 & 40.923 .603 & $-0,98$ \\
\hline 25 & Servicios destinados a la venta & 2.719 .056 & 2.608 .181 & 22.723 .370 & 22.371 .689 & $-1,55$ \\
\hline 26 & Servicios no destinados a la venta & 12.250 .089 & 12.043 .061 & 13.072 .071 & 12.854 .010 & $-1,67$ \\
\hline 27 & Trabajo & 0 & 0 & 47.273 .329 & 46.601 .605 & $-1,42$ \\
\hline 28 & Capital & 0 & 0 & 52.414 .709 & 51.644 .948 & $-1,47$ \\
\hline \multirow[t]{2}{*}{29} & Consumo & 27.103 .250 & 27.103 .250 & 126.791 .288 & 125.349 .803 & $-1,14$ \\
\hline & Total & 125.727.719 & 124.099 .024 & 532.341 .763 & 525.041 .909 & $-1,37$ \\
\hline
\end{tabular}

Fuente: Elaboración propia. 
En la Tabla 5 podemos observar el vector Z, el cual contiene las inyecciones totales de renta que cada una de las cuentas denominadas endógenas recibe del conjunto de cuentas denominadas exógenas; el vector ZsinFONDOS al cual se le ha suprimido las inyecciones procedentes de Fondos Estructurales; el vector $\mathrm{Y}$, que contiene los outputs totales de las cuentas endógenas en un escenario con Fondos; el vector YsinFONDOS que contiene el total de outputs de las cuentas endógenas sin haber recibido Fondos y en la última columna podemos apreciar el porcentaje de variación tras la eliminación de dichos Fondos.

Podemos observar como las ramas de actividad que se ven afectadas en mayor medida por la detracción de los Fondos son: Ganadería (2) con un 4,40\% de disminución de output, Pesca (3) con un 4,06\%, y Maquinaria (16) con un $2,43 \%$. Por el contrario, las ramas de actividad que menos afectadas se ven por dicha eliminación de Fondos son: Refino de petróleo (6), con un descenso de output de $0,87 \%$ Vehículos (17) con un $0,94 \%$ y Otros servicios (24), con un descenso de output de $0,98 \%$.

A continuación pasaremos al segundo escenario en el que como hemos comentado anteriormente, a la economía de Andalucía sin Fondos se le inyectan todos los Fondos Estructurales recibidos correspondientes al año 2005, para realizar un análisis de la aportación de cada Fondo.

\subsection{Estimación del impacto de los Fondos FEDER sobre la economía de Andalucía en el año 2005}

A continuación se puede observar la Tabla 6, en la que se recoge el reparto de los recursos procedentes del FEDER entre las distintas ramas de actividad para el año objeto de estudio; dicho reparto se ha realizado del mismo modo que el reparto del conjunto de de los recursos, analizados en el epígrafe anterior.

\section{Tabla 6}

Asignación de los Fondos FEDER año 2005 a las ramas de actividad (miles de euros)

\begin{tabular}{|c|c|c|}
\hline & Ramas de Actividad & Fondo FEDER \\
\hline 1 & Agricultura & 12.638 \\
\hline 2 & Ganadería & 9.650 \\
\hline 3 & Pesca & 17.058 \\
\hline 4 & Extracción de productos energéticos & 1.097 \\
\hline 5 & Resto de extractivas & 1.159 \\
\hline 6 & Refino de petróleo y tratamiento de residuos nucleares & 15.514 \\
\hline 7 & Producción y distribución de energía eléctrica & 5.932 \\
\hline 8 & Producción y distribución de gas, vapor de agua y agua caliente & 5.847 \\
\hline 9 & Captación, depuración y distribución de agua & 1.194 \\
\hline 10 & Alimentación & 15.462 \\
\hline
\end{tabular}


Tabla 6 (continuación)

Asignación de los Fondos FEDER año 2005 a las ramas de actividad (miles de euros)

\begin{tabular}{|c|c|c|}
\hline & Ramas de Actividad & Fondo FEDER \\
\hline 11 & Textil y piel & 6.903 \\
\hline 12 & Elaborados de madera & 5.931 \\
\hline 13 & Químicas & 12.534 \\
\hline 14 & Minería y siderurgia & 8.359 \\
\hline 15 & Elaborados metálicos & 7.839 \\
\hline 16 & Maquinaria & 160.270 \\
\hline 17 & Vehículos & 10.928 \\
\hline 18 & Materiales de construcción & 6.324 \\
\hline 19 & Transporte & 7.454 \\
\hline 20 & Otras manufacturas & 13.031 \\
\hline 21 & Construcción & 574.155 \\
\hline 22 & Resto de comercio & 57.145 \\
\hline 23 & Transporte y comunicaciones & 12.863 \\
\hline 24 & Otros servicios & 73.153 \\
\hline 25 & Servicios destinados a la venta & 32.826 \\
\hline \multirow[t]{2}{*}{26} & Servicios no destinados a la venta & 169.967 \\
\hline & Total & 1.245 .233 \\
\hline
\end{tabular}

Fuente: Elaboración propia a partir de Lima, Cardenete y Usabiaga (2010).

La Tabla 6 muestra las cantidades que vamos a añadir al vector de cuentas exógenas sin Fondos para obtener el vector sin Fondos con la suma del FEDER y aplicando el modelo de multiplicadores se obtendrá el vector YsinFONDOS + FEDER.

A continuación, en la Tabla 7, se muestra el aumento de las cuentas endógenas (YsinFONDOS + FEDER - YsinFONDOS) así como el aumento porcentual de output en cada cuenta a causa de la inyección del FEDER.

En dicha tabla se puede observar como el output total aumenta 5.611.795 miles de euros, es decir, un 1,06\% de aumento. Las ramas de actividad con un mayor aumento de output son: Pesca (2) con un 2,72\% de incremento, Maquinaria (16) con un $2,26 \%$, Construcción (21) con un $1,95 \%$ y Materiales de construcción (18) con un 1,71\%. Por el contrario las ramas de actividad menos afectadas por la inyección de FEDER son: Agricultura (1) con un 0,57\%, Alimentación (10) con un $0,64 \%$, Refino de Petróleo (6) con un 0,66\% y Vehículos (17) con un $0,69 \%$ de incremento.

Algunas cuentas ven aumentado su output en mayor medida que otras que reciben más financiación del FEDER; es el caso de Materiales de construcción (18), que recibe menos financiación que Servicios no destinados a la venta (26) 
y su output crece en mayor cuantía, debido a que su estructura productiva es capaz de captar y aprovechar de mejor forma esa inyección de renta.

Tabla 7

Aumento del output total tras inyectar los Fondos FEDER 2005

(miles de euros)

\begin{tabular}{|c|c|c|c|c|c|}
\hline & Ramas de Actividad & YsinFONDOS & $\begin{array}{l}\text { YsinFONDOS } \\
\text { + FEDER }\end{array}$ & $\begin{array}{c}\text { YsinFONDOS } \\
\text { + FEDER - } \\
\text { YsinFONDOS }\end{array}$ & $\begin{array}{l}\text { Aumento } \\
(\%)\end{array}$ \\
\hline 1 & Agricultura & 9.317 .521 & 9.370 .750 & 53.228 & 0,57 \\
\hline 2 & Ganadería & 2.397 .902 & 2.421 .915 & 24.013 & 0,99 \\
\hline 3 & Pesca & 806.957 & 829.525 & 22.567 & 2,72 \\
\hline 4 & Extracción de productos energéticos & 6.270 .393 & 6.321 .427 & 51.034 & 0,81 \\
\hline 5 & Resto de extractivas & 2.397 .830 & 2.431 .126 & 33.296 & 1,37 \\
\hline 6 & $\begin{array}{l}\text { Refino de petróleo y tratamiento de } \\
\text { residuos nucleares }\end{array}$ & 13.675 .231 & 13.765 .954 & 90.722 & 0,66 \\
\hline 7 & $\begin{array}{l}\text { Producción y distribución de energía } \\
\text { eléctrica }\end{array}$ & 3.321 .897 & 3.361 .167 & 39.270 & 1,17 \\
\hline 8 & $\begin{array}{l}\text { Producción y distribución de gas, } \\
\text { vapor de agua y agua caliente }\end{array}$ & 1.127 .096 & 1.145 .641 & 18.545 & 1,62 \\
\hline 9 & $\begin{array}{l}\text { Captación, depuración y distribución } \\
\text { de agua }\end{array}$ & 1.061 .905 & 1.073 .304 & 11.399 & 1,06 \\
\hline 10 & Alimentación & 21.835 .842 & 21.977 .201 & 141.359 & 0,64 \\
\hline 11 & Textil y piel & 4.006 .823 & 4.044 .001 & 37.178 & 0,92 \\
\hline 12 & Elaborados de madera & 3.580 .446 & 3.621 .854 & 41.408 & 1,14 \\
\hline 13 & Químicas & 9.619 .169 & 9.692 .406 & 73.237 & 0,76 \\
\hline 14 & Minería y siderurgia & 6.051 .664 & 6.107 .879 & 56.215 & 0,92 \\
\hline 15 & Elaborados metálicos & 4.419 .069 & 4.480 .919 & 61.850 & 1,38 \\
\hline 16 & Maquinaria & 12.117 .231 & 12.397 .976 & 280.745 & 2,26 \\
\hline 17 & Vehículos & 6.776 .931 & 6.824 .256 & 47.325 & 0,69 \\
\hline 18 & Materiales de construcción & 6.479 .280 & 6.592 .132 & 112.851 & 1,71 \\
\hline 19 & Transporte & 1.819 .241 & 1.835 .218 & 15.977 & 0,87 \\
\hline 20 & Otras manufacturas & 7.052 .847 & 7.114 .766 & 61.919 & 0,87 \\
\hline 21 & Construcción & 45.109 .582 & 46.007 .339 & 897.757 & 1,95 \\
\hline 22 & Resto de comercio & 37.888 .541 & 38.270 .172 & 381.631 & 1,00 \\
\hline 23 & Transporte y comunicaciones & 18.162 .850 & 18.349 .558 & 186.709 & 1,02 \\
\hline 24 & Otros servicios & 40.923 .603 & 41.231 .651 & 308.047 & 0,75 \\
\hline 25 & Servicios destinados a la venta & 22.371 .689 & 22.587 .307 & 215.618 & 0,95 \\
\hline 26 & Servicios no destinados a la venta & 12.854 .010 & 13.032 .560 & 178.550 & 1,37 \\
\hline 27 & Trabajo & 46.601 .605 & 47.128 .416 & 526.811 & 1,12 \\
\hline 28 & Capital & 51.644 .948 & 52.202 .808 & 557.860 & 1,07 \\
\hline \multirow[t]{2}{*}{29} & Consumo & 125.349 .803 & 126.434 .475 & 1.084 .671 & 0,86 \\
\hline & Total & 525.041 .909 & 530.653 .704 & 5.611 .795 & 1,06 \\
\hline
\end{tabular}

Fuente: Elaboración propia. 


\subsection{Estimación del impacto de los Fondos FEOGA-O sobre la economía de Andalucía en el año 2005}

De la misma forma que el caso anterior, en la Tabla 8 se puede observar el reparto de los recursos procedentes del FEOGA-O entre las distintas ramas de actividad para el año objeto de estudio.

\section{Tabla 8}

Asignación de los Fondos FEOGA-O año 2005 a las ramas de actividad (miles de euros)

\begin{tabular}{|c|c|c|}
\cline { 2 - 3 } \multicolumn{1}{c|}{} & Ramas de Actividad & Fondo FEOGA-O \\
\hline 1 & Agricultura & 50.072 \\
\hline 2 & Ganadería & 71.934 \\
\hline 3 & Pesca & 4.683 \\
\hline 10 & Alimentación & 16.707 \\
\hline 22 & Resto de comercio & 16.267 \\
\hline 24 & Otros servicios & 7.816 \\
\hline & Total & $\mathbf{1 6 7 . 4 8 0}$ \\
\hline
\end{tabular}

Fuente: Elaboración propia a partir de Lima, Cardenete y Usabiaga (2010).

La Tabla 8 muestra las cantidades que vamos a añadir al vector de cuentas exógenas sin Fondos para obtener el vector sin Fondos con la suma del FEOGA-O y aplicando el modelo de multiplicadores se obtendrá el vector YsinFONDOS + FEOGA-O.

En la tabla que aparece a continuación se muestra el aumento de las cuentas endógenas (YsinFONDOS + FEOGA-O - YsinFONDOS) así como el aumento porcentual de output en cada cuenta a causa de la inyección del FEOGA-O.

En la Tabla 9 se puede observar como el output total aumenta 761.919 miles de euros, aproximadamente un $0,14 \%$ de aumento medio. Las ramas de actividad con un mayor aumento de output son: Ganadería (2) con un 3,18\%, Agricultura (1) con un $0,72 \%$, Pesca (3) con un $0,71 \%$, Captación, depuración y distribución de agua (9) y Alimentación (10) con un $0,25 \%$ de incremento cada una. Por el contrario las ramas de actividad menos afectadas por la inyección de FEOGA-O son: Servicios no destinados a la venta (26) con un 0,01 , Construcción (21) con un $0,02 \%$, Materiales de construcción (18), Minería y Siderurgia (14) y Resto de extractivas (5), todas ellas con un $0,04 \%$ de incremento de output.

Resulta significativo si comparamos los resultados obtenidos con los datos de inyección de Fondos FEOGA-O de la Tabla 8, como dentro de las ramas más beneficiadas se encuentran algunas que no reciben Fondos FEOGA-O, siendo 
ésto constatable en la cuenta Captación, depuración y distribución de agua (9), que aún no recibiendo inyección de Fondos FEOGA-O se encuentra entre las cuatro primeras ramas de actividad con un mayor aumento de output, pudiendo ser este efecto atribuido, a la relación directa de esta cuenta con las cuentas que mayor montante de FEOGA-O reciben.

Tabla 9

Aumento del output total tras inyectar los Fondos FEOGA-O 2005 (miles de euros)

\begin{tabular}{|c|c|c|c|c|c|}
\hline & Ramas de Actividad & YsinFONDOS & $\begin{array}{l}\text { YsinFONDOS } \\
+ \text { FEOGA-O }\end{array}$ & $\begin{array}{l}\text { YsinFONDOS } \\
\text { + FEOGA-O - } \\
\text { YsinFONDOS }\end{array}$ & $\begin{array}{c}\text { Aumento } \\
(\%)\end{array}$ \\
\hline 1 & Agricultura & 9.317 .521 & 9.385 .462 & 67.941 & 0,72 \\
\hline 2 & Ganadería & 2.397 .902 & 2.476 .622 & 78.720 & 3,18 \\
\hline 3 & Pesca & 806.957 & 812.710 & 5.753 & 0,71 \\
\hline 4 & Extracción de productos energéticos & 6.270 .393 & 6.275 .747 & 5.354 & 0,09 \\
\hline 5 & Resto de extractivas & 2.397 .830 & 2.398 .769 & 939 & 0,04 \\
\hline 6 & $\begin{array}{l}\text { Refino de petróleo y tratamiento de } \\
\text { residuos nucleares }\end{array}$ & 13.675 .231 & 13.685 .528 & 10.296 & 0,08 \\
\hline 7 & $\begin{array}{l}\text { Producción y distribución de energía } \\
\text { eléctrica }\end{array}$ & 3.321 .897 & 3.326 .915 & 5.017 & 0,15 \\
\hline 8 & $\begin{array}{l}\text { Producción y distribución de gas, } \\
\text { vapor de agua y agua caliente }\end{array}$ & 1.127 .096 & 1.128 .597 & 1.501 & 0,13 \\
\hline 9 & $\begin{array}{l}\text { Captación, depuración y distribución } \\
\text { de agua }\end{array}$ & 1.061 .905 & 1.064 .605 & 2.700 & 0,25 \\
\hline 10 & Alimentación & 21.835 .842 & 21.890 .177 & 54.335 & 0,25 \\
\hline 11 & Textil y piel & 4.006 .823 & 4.011 .199 & 4.376 & 0,11 \\
\hline 12 & Elaborados de madera & 3.580 .446 & 3.583 .896 & 3.450 & 0,10 \\
\hline 13 & Químicas & 9.619 .169 & 9.628 .727 & 9.558 & 0,10 \\
\hline 14 & Minería y siderurgia & 6.051 .664 & 6.054 .018 & 2.355 & 0,04 \\
\hline 15 & Elaborados metálicos & 4.419 .069 & 4.422 .714 & 3.645 & 0,08 \\
\hline 16 & Maquinaria & 12.117 .231 & 12.125 .045 & 7.814 & 0,06 \\
\hline 17 & Vehículos & 6.776 .931 & 6.782 .266 & 5.335 & 0,08 \\
\hline 18 & Materiales de construcción & 6.479 .280 & 6.481 .815 & 2.535 & 0,04 \\
\hline 19 & Transporte & 1.819 .241 & 1.820 .301 & 1.060 & 0,06 \\
\hline 20 & Otras manufacturas & 7.052 .847 & 7.058 .501 & 5.654 & 0,08 \\
\hline 21 & Construcción & 45.109 .582 & 45.119 .258 & 9.676 & 0,02 \\
\hline 22 & Resto de comercio & 37.888 .541 & 37.955 .600 & 67.059 & 0,18 \\
\hline 23 & Transporte y comunicaciones & 18.162 .850 & 18.184 .141 & 21.292 & 0,12 \\
\hline 24 & Otros servicios & 40.923 .603 & 40.964 .663 & 41.060 & 0,10 \\
\hline 25 & Servicios destinados a la venta & 22.371 .689 & 22.396 .783 & 25.094 & 0,11 \\
\hline 26 & Servicios no destinados a la venta & 12.854 .010 & 12.854 .741 & 731 & 0,01 \\
\hline 27 & Trabajo & 46.601 .605 & 46.661 .816 & 60.211 & 0,13 \\
\hline 28 & Capital & 51.644 .948 & 51.744 .073 & 99.125 & 0,19 \\
\hline \multirow[t]{2}{*}{29} & Consumo & 125.349 .803 & 125.509 .139 & 159.335 & 0,13 \\
\hline & Total & 525.041 .909 & 525.803 .827 & 761.919 & 0,14 \\
\hline
\end{tabular}

Fuente: Elaboración propia. 


\subsection{Estimación del impacto de los Fondos FSE sobre la economía de Andalucía en el año 2005}

De la misma forma que los casos anteriores, en la Tabla 10 se puede observar el reparto de los recursos procedentes del FSE entre las distintas ramas de actividad para el año objeto de estudio.

Tabla 10

Asignación de los Fondos FSE año 2005 a las ramas de actividad (miles de euros)

\begin{tabular}{|c|c|c|}
\hline & Ramas de Actividad & Fondo FSE \\
\hline 1 & Agricultura & 5.176 \\
\hline 2 & Ganadería & 4.680 \\
\hline 3 & Pesca & 4.784 \\
\hline 4 & Extracción de productos energéticos & 3.007 \\
\hline 5 & Resto de extractivas & 3.177 \\
\hline 6 & Refino de petróleo y tratamiento de residuos nucleares & 7.396 \\
\hline 7 & Producción y distribución de energía eléctrica & 2.828 \\
\hline 8 & Producción y distribución de gas, vapor de agua y agua caliente & 2.788 \\
\hline 9 & Captación, depuración y distribución de agua & 12 \\
\hline 10 & Alimentación & 4.411 \\
\hline 11 & Textil y piel & 3.060 \\
\hline 12 & Elaborados de madera & 3.022 \\
\hline 13 & Químicas & 5.976 \\
\hline 14 & Minería y siderurgia & 4.742 \\
\hline 15 & Elaborados metálicos & 3.590 \\
\hline 16 & Maquinaria & 2.383 \\
\hline 17 & Vehículos & 5.210 \\
\hline 18 & Materiales de construcción & 3.223 \\
\hline 19 & Transporte & 3.554 \\
\hline 20 & Otras manufacturas & 3.567 \\
\hline 21 & Construcción & 2.464 \\
\hline 22 & Resto de comercio & 7.287 \\
\hline 23 & Transporte y comunicaciones & 3.087 \\
\hline 24 & Otros servicios & 11.447 \\
\hline 25 & Servicios destinados a la venta & 78.049 \\
\hline \multirow[t]{2}{*}{26} & Servicios no destinados a la venta & 37.062 \\
\hline & Total & 215.983 \\
\hline
\end{tabular}

Fuente: Elaboración propia a partir de Lima, Cardenete y Usabiaga (2010).

La Tabla 10 muestra las cantidades que vamos a añadir al vector de cuentas exógenas sin Fondos para obtener el vector sin Fondos con la suma del FSE y 
aplicando el modelo de multiplicadores se obtendrá el vector YsinFONDOS + FSE.

En la tabla que aparece a continuación se muestra el aumento de las cuentas endógenas (YsinFONDOS + FSE - YsinFONDOS) así como el aumento porcentual de output en cada cuenta a causa de la inyección del FSE.

Tabla 11

Aumento del output total tras inyectar los Fondos FSE 2005

(miles de euros)

\begin{tabular}{|c|c|c|c|c|c|}
\hline & Ramas de Actividad & YsinFONDOS & $\begin{array}{l}\text { YsinFONDOS } \\
+ \text { FSE }\end{array}$ & $\begin{array}{c}\text { YsinFONDOS } \\
\text { + FSE - } \\
\text { YsinFONDOS }\end{array}$ & $\begin{array}{l}\text { Aumento } \\
(\%)\end{array}$ \\
\hline 1 & Agricultura & 9.317 .521 & 9.330 .777 & 13.256 & 0,14 \\
\hline 2 & Ganadería & 2.397 .902 & 2.405 .441 & 7.539 & 0,31 \\
\hline 3 & Pesca & 806.957 & 812.759 & 5.802 & 0,71 \\
\hline 4 & Extracción de productos energéticos & 6.270 .393 & 6.284 .798 & 14.405 & 0,23 \\
\hline 5 & Resto extractivas & 2.397 .830 & 2.403 .962 & 6.132 & 0,26 \\
\hline 6 & $\begin{array}{l}\text { Refino de petróleo y tratamiento residuos } \\
\text { nucleares }\end{array}$ & 13.675 .231 & 13.694 .913 & 19.682 & 0,14 \\
\hline 7 & $\begin{array}{l}\text { Producción y distribución de energía } \\
\text { eléctrica }\end{array}$ & 3.321 .897 & 3.330 .608 & 8.710 & 0,26 \\
\hline 8 & $\begin{array}{l}\text { Producción y distribución de gas, vapor } \\
\text { de agua y agua caliente }\end{array}$ & 1.127.096 & 1.132 .319 & 5.222 & 0,46 \\
\hline 9 & $\begin{array}{l}\text { Captación, depuración y distribución de } \\
\text { agua }\end{array}$ & 1.061 .905 & 1.063 .779 & 1.874 & 0,18 \\
\hline 10 & Alimentación & 21.835 .842 & 21.863 .739 & 27.897 & 0,13 \\
\hline 11 & Textil y piel & 4.006 .823 & 4.015 .763 & 8.940 & 0,22 \\
\hline 12 & Elaborados de madera & 3.580 .446 & 3.588 .173 & 7.727 & 0,22 \\
\hline 13 & Químicas & 9.619 .169 & 9.635 .156 & 15.987 & 0,17 \\
\hline 14 & Minería y siderurgia & 6.051 .664 & 6.062 .009 & 10.345 & 0,17 \\
\hline 15 & Elaborados metálicos & 4.419 .069 & 4.426 .282 & 7.214 & 0,16 \\
\hline 16 & Maquinaria & 12.117 .231 & 12.130 .493 & 13.262 & 0,11 \\
\hline 17 & Vehículos & 6.776 .931 & 6.788 .666 & 11.735 & 0,17 \\
\hline 18 & Materiales de construcción & 6.479 .280 & 6.486 .465 & 7.184 & 0,11 \\
\hline 19 & Transporte & 1.819 .241 & 1.825 .055 & 5.814 & 0,32 \\
\hline 20 & Otras manufacturas & 7.052 .847 & 7.064 .197 & 11.351 & 0,16 \\
\hline 21 & Construcción & 45.109 .582 & 45.131 .838 & 22.256 & 0,05 \\
\hline 22 & Resto de comercio & 37.888 .541 & 37.953 .143 & 64.602 & 0,17 \\
\hline 23 & Transporte y comunicaciones & 18.162 .850 & 18.192 .081 & 29.231 & 0,16 \\
\hline 24 & Otros servicios & 40.923 .603 & 40.978 .871 & 55.268 & 0,13 \\
\hline 25 & Servicios destinados a la venta & 22.371 .689 & 22.482 .658 & 110.969 & 0,49 \\
\hline 26 & Servicios no destinados a la venta & 12.854 .010 & 12.892 .791 & 38.781 & 0,30 \\
\hline 27 & Trabajo & 46.601 .605 & 46.686 .307 & 84.702 & 0,18 \\
\hline 28 & Capital & 51.644 .948 & 51.757 .725 & 112.776 & 0,22 \\
\hline \multirow[t]{2}{*}{29} & Consumo & 125.349 .803 & 125.547 .282 & 197.478 & 0,16 \\
\hline & Total & 525.041 .909 & 525.968 .050 & 926.141 & 0,18 \\
\hline
\end{tabular}

Fuente: Elaboración propia. 
En la Tabla 11 se puede observar como el output total aumenta $926.141 \mathrm{mi}-$ les de euros, aproximadamente un $0,18 \%$ de aumento. Las ramas de actividad con un mayor aumento de output son: Pesca (3) con un $0,71 \%$, Servicios destinados a la venta (25) con un $0,49 \%$, Producción y distribución de gas, vapor de agua y agua caliente (8) con un $0,46 \%$, Transporte (19) con un $0,32 \%$, Ganadería (2) con un $0,31 \%$ y Servicios no destinados a la venta (26) con un $0,30 \%$. Por el contrario las ramas de actividad menos afectadas por la inyección de FSE son: Construcción (21) con un $0,05 \%$, Materiales de construcción (18) y Maquinaria (16) con un $0,11 \%$ de incremento de output.

$\mathrm{Al}$ igual que ocurría con el FEDER, cuentas que reciben un menor porcentaje que otras resultan más beneficiadas debido a que su estructura productiva es capaz de absorber esta inyección de Fondos de una mejor forma; en concreto ocurre con la cuenta Pesca (3) que, aún recibiendo menos Fondos, resulta más beneficiada que Servicios destinados a la venta (26) que es una de las que más inyección de Fondos FSE recibe de entre todas la ramas de actividad.

\section{CONCLUSIONES}

En este trabajo se pretende identificar los sectores productivos andaluces más beneficiados por la recepción de Fondos Estructurales provenientes de la Unión Europea. Para ello se ha utilizado la matriz de contabilidad social del año 2005 y se ha aplicado un modelo de multiplicadores lineales. Los resultados obtenidos clasifican los sectores de la economía andaluza según su capacidad para absorber las inyecciones exógenas de renta procedentes de dichos Fondos, produciendo así cambios estructurales en los sectores clave que serán los encargados de tirar del crecimiento de la economía regional.

Los resultados obtenidos ponen de manifiesto la necesidad de la región de la percepción de estos Fondos. Podemos destacar tras el estudio realizado, que las variaciones de output son bastante significativas.

En el caso del escenario base, en el que se simulaba una retirada total de los Fondos Estructurales, observamos que la repercusión sobre el output es de un decremento medio de 1,4\%, siendo, Ganadería (2), Pesca (3) y Maquinaria (16), las ramas de actividad que se ven afectadas en mayor medida por la eliminación de los Fondos.

Por el contrario, si observamos los datos obtenidos en el segundo escenario, en el que se ha procedido a realizar las inyecciones de cada Fondo, partiendo del escenario en el que la economía andaluza tiene aplicada la retirada total de Fondos del año 2005, los aumentos medios de output en las ramas de actividad han sido significativos también; en el caso de la inyección del Fondo FEDER el aumento de output ha sido de 5.611.795 miles de euros, es decir, un aumento porcentual de 1,06\%, siendo las ramas de actividad con una mayor capacidad de absorción: Pesca (3), Maquinaria (16) y Construcción (21) entre otras. En el 
caso de la inyección del Fondo FEOGA-O el aumento de output ha sido de 761.919 miles de euros, es decir, un aumento porcentual de $0,14 \%$, siendo el sector primario (Ganadería (2) Agricultura (1) y Pesca (3)) el que cuenta con una mayor capacidad de absorción. Por último, si observamos la inyección del Fondo FSE el aumento de output es de 926.141 miles de euros, es decir, un aumento porcentual medio de $0,18 \%$, siendo Pesca (3) y Servicios destinados a la venta (25) las ramas de actividad con una mayor capacidad de absorción.

Podemos concluir destacando que los Fondos Estructurales incentivan el desarrollo de la región andaluza mediante las inversiones realizadas por la Política Regional Europea, que como hemos podido observar no sólo afecta a los sectores en lo que se invierte de manera directa, sino que acaban influyendo en todos los sectores de la economía.

\section{REFERENCIAS BIBLIOGRÁFICAS}

CÁMARA, A. y MARCOS, M.A. (2009): "Análisis del impacto de los Fondos Europeos 2000-2006 en la Comunidad de Madrid a partir de la matriz de contabilidad social del año 2000", Investigaciones Regionales, 13, pp. 7192.

CARDENETE, M.A. (2004):" Evaluación de una reducción de las cuotas empresariales a la Seguridad Social a nivel regional a través de un Modelo de Equilibrio General Aplicado: el caso de Andalucía". Estudios de Economía Aplicada, 22(1), pp. 57-71.

CARDENETE, M.A., FUENTES, P. y POLO, C. (2010): "Análisis de sectores clave a partir de la matriz de contabilidad social de Andalucía para el año 2000", Revista de Estudios Regionales, 88, pp. 15-44.

CARDENETE, M. A., y LLOP, M. (2005). "Modelos multisectoriales de equilibrio general aplicado en España: Una revisión". Estudios de Economía Aplicada, 23(2), pp. 385-404.

COMISIÓN EUROPEA (1986): "Sexto informe periódico sobre la situación socioeconómica y desarrollo de las regiones de la Unión Europea", Bruselas.

CONSEJERÍA DE ECONOMÍA Y HACIENDA (2001): "Plan de desarrollo regional, marco comunitario de apoyo y programa operativo integrado de Andalucía 2000-2006", Sevilla.

DEFOURNEY, J. y THORBECKE, E. (1984): "Structural Path Analysis and Multiplier Decomposition within a Social Accounting Matrix framework", The Economic Journal, 94, pp.111-136.

DE LA FUENTE, A. (2005): "El impacto de la reducción de las ayudas estructurales europeas: una primera aproximación", Documento de Trabajo D-2005- 
04, Dirección General de Presupuestos, Ministerio de Economía y Hacienda.

DONES, M. y PÉREZ, J. (2002): "Evaluación de los efectos macroeconómicos de los Fondos Estructurales y los Fondos de Cohesión (1995-1999) mediante Tablas Input-Output regionales integradas", Cuadernos del Fondo de Investigación Richard Stone,2, Instituto L.R. Klein-Centro Stone.

FUENTES, P. (2012):" Una estimación de la Matriz de Contabilidad Social de Andalucía de 2005 a precios básicos" mimeo.

GHOSH, A. (1958): "Input-Output Approach in Allocation System”, Económica, 25, 58-64.

LEONTIEF, W. (1941): The Structure of American Economy, 1919-1924: an Empiriocal Application of Equilibrium Analysis, Harvard Univ. Press, Cambridge, Mass.

LIMA, M. C. y CARDENETE, M. A. (2005): "Análisis de impacto de los Fondos FEDER recibidos por una economía regional: un enfoque a través de matrices de contabilidad social", Presupuesto y Gasto Público, 40(3), pp. 113131.

LIMA, M. C. y CARDENETE, M. A. (2008): "The Impact of the European Structural Funds in the south of Spain: A CGE Approach", European Planning Studies, 16(10), pp.1445-1457.

LIMA, M. C., CARDENETE, M. A. y USABIAGA, C. (2010): "Andalucía y el MAC 2000-2006: Una evaluación de los Fondos Estructurales recibidos", Papeles de Economía Española, 123, pp. 102-118.

LLANO, C. (2004). "The interregional trade in the context of a multirregional input-output model for Spain". Estudios de economía aplicada, 22(3), pp. 539-576.

LLOP, M. (2007). "Descomposición de los cambios de multiplicadores en una matriz de contabilidad social: un ejemplo para la economía catalana". Estudios de economía aplicada, 25, pp. 743-758.

LÓPEZ, L. A., MONSALVE, F. y ZAFRILLA, J.E. (2011): “Análisis Input - Output de la eficacia de la Política Europea de Desarrollo Rural 2007 - 2013. Propuesta metodológica y resultados para Castilla- La Mancha", Estudios de Economía Aplicada, 29(1), pp. 223-246.

MÁRQUEZ, M.A., RAMAJO, J. y De MIGUEL, F.J. (2010): "Evaluación de los efectos económicos de los Fondos Estructurales del periodo 2000-2006 sobre Extremadura", Papeles de Economía Española, 123, pp. 191-205.

MONROBEL, J. R., CÁMARA, A., MARCOS, M. A. (2012): "Modeling European Regional Policy 2007-2013: Applied General Equilibrium Analysis of the Economic Impact on the Madrid Region", European Planning Studies, http://dx.doi.org/10.1080/09654313.2012.722925.

MORILLAS, A., MONICHE, L. y MARCOS, J. (1999): "Evaluación de los efectos de los Fondos Estructurales en la economía andaluza", Estudios Regionales, 54, pp. 225-249. 
MORILLAS, A., MONICHE, L. y MARCOS, J. (2004): "Efectos ultra frontera y convergencia regional: una reflexión a partir del MAC 94-99 en Andalucía", http://campusvirtual.uma.es/morillas/Fondos.pdf.

MURILLO, E. (2005): La política regional europea: un análisis de sus efectos sobre la economía andaluza: período, 1994-2001. Madrid: Instituto de Estudios Fiscales.

MURILLO, E. (2007): Análisis de los efectos económicos de la Política Regional Europea en Andalucía, Sevilla: Consejo Económico y Social de Andalucía.

POLO, C., ROLAND-HOLST, D. y SANCHO, F. (1990): "Descomposición de Multiplicadores en un Modelo Multisectorial: una Aplicación al Caso Español", Investigaciones Económicas, 15 (1), pp.53-69.

PYATT, G. y ROUND, J.I. (1979): "Accounting and fixed price multipliers in a Social Accounting Matrix framework", The Economic Journal, 89 (356), pp. 850-873.

SOSVILLA, S. (2003): "Canarias y los Fondos Estructurales Europeos", Documento de Trabajo 2003-28, FEDEA.

SOSVILLA, S. (2007): "La economía española y la política de cohesión europea", ICE, Revista de Economía, 837, pp. 211-230.

SOSVILLA, S., BAJO, O. y DÍAZ, C. (2003): "Efectividad de la política regional comunitaria: el caso de Castilla-La Mancha", Papeles de Economía Española 2003-25, 107, pp. 243-255.

SOSVILLA, S. y GARCÍA, E. (2006): "Efectos de las ayudas europeas sobre la economía madrileña, 2007-2013: Un análisis basado en el modelo Hermin". Documento de Trabajo 2006-07, FEDEA.

SOSVILLA, S. y HERCE, J. A. (2003): "Efectos de las ayudas europeas sobre la economía madrileña, 1990-2006: Un análisis basado en el modelo Hermin". Documento de Trabajo 2003-29, FEDEA.

SOSVILLA, S. y MURILLO, E. (2005): "Efectos de oferta sobre la economía andaluza de las ayudas procedentes de los Fondos Estructurales destinadas a infraestructuras: el Marco de Apoyo Comunitario 1994-1999", Investigaciones Regionales, 6, pp. 91-124.

STONE, R. (1962): A Social Accounting Matrix for 1960: A Programme for Growth, Chapman and Hall Ltd. (Eds.), London. 


\section{APÉNDICE}

Matriz de Contabilidad Social de Andalucía del año 2005 a precios básicos

\begin{tabular}{|c|c|c|c|c|c|c|c|c|c|c|}
\hline & 1 & 2 & 3 & 4 & 5 & 6 & 7 & 8 & 9 & 10 \\
\hline 1 & 533378 & 79195 & 79 & 0 & 27 & 4604 & 3 & 3 & 2092 & 4010089 \\
\hline 2 & 61599 & 85612 & 0 & 0 & 13 & 890 & 0 & 26 & 54 & 1214406 \\
\hline 3 & 301 & 10 & 10443 & 0 & 21 & 0 & 0 & 1 & 72 & 127564 \\
\hline 4 & 17 & 6 & 0 & 10066 & 4 & 5168407 & 330750 & 628555 & 179 & 3604 \\
\hline 5 & 50 & 586 & 163 & 1 & 29418 & 29922 & 267 & 204 & 451 & 10208 \\
\hline 6 & 271252 & 25488 & 47553 & 3440 & 60867 & 2361876 & 218343 & 99769 & 6444 & 87643 \\
\hline 7 & 93435 & 18387 & 1631 & 2417 & 21728 & 61912 & 142445 & 30721 & 31563 & 137108 \\
\hline 8 & 1766 & 146 & 1820 & 201 & 4074 & 28557 & 459036 & 43833 & 1097 & 51513 \\
\hline 9 & 157022 & 14238 & 6 & 31 & 819 & 7715 & 4071 & 559 & 63404 & 24157 \\
\hline 10 & 83084 & 362951 & 16642 & 0 & 445 & 452 & 0 & 13 & 1515 & 4574444 \\
\hline 11 & 15414 & 1918 & 7796 & 32 & 499 & 92 & 131 & 15 & 155 & 11280 \\
\hline 12 & 19934 & 6542 & 2811 & 46 & 2393 & 4159 & 1899 & 597 & 1096 & 274508 \\
\hline 13 & 386660 & 37449 & 7341 & 2202 & 35058 & 361853 & 4406 & 557 & 18971 & 99692 \\
\hline 14 & 12 & 527 & 0 & 36 & 1823 & 1054 & 844 & 128 & 2500 & 2401 \\
\hline 15 & 169714 & 22581 & 18 & 19 & 43269 & 3644 & 23654 & 832 & 3279 & 67190 \\
\hline 16 & 60056 & 18157 & 5561 & 2593 & 48553 & 84688 & 127603 & 16360 & 40892 & 186491 \\
\hline 17 & 1389 & 1466 & 0 & 0 & 9741 & 2 & 52 & 4 & 517 & 8391 \\
\hline 18 & 11983 & 7270 & 0 & 3 & 6038 & 3231 & 1950 & 1458 & 6355 & 267841 \\
\hline 19 & 653 & 7690 & 18470 & 384 & 66 & 0 & 0 & 0 & 0 & 496 \\
\hline 20 & 87339 & 4386 & 1200 & 166 & 5011 & 21576 & 1906 & 1888 & 3478 & 205936 \\
\hline 21 & 43978 & 55735 & 0 & 33 & 14445 & 9742 & 43630 & 5329 & 64761 & 61360 \\
\hline 22 & 305093 & 109235 & 32274 & 333 & 22553 & 59882 & 15943 & 5788 & 31252 & 349679 \\
\hline 23 & 194790 & 46835 & 13217 & 3624 & 129611 & 264213 & 44306 & 77966 & 19524 & 528583 \\
\hline 24 & 232928 & 132156 & 6233 & 1512 & 39174 & 345235 & 155810 & 55300 & 59874 & 692485 \\
\hline 25 & 15371 & 18220 & 770 & 3749 & 16561 & 108523 & 17918 & 10222 & 21039 & 127170 \\
\hline 26 & 1401 & 1458 & 347 & 0 & 516 & 2275 & 236 & 10 & 58 & 7517 \\
\hline 27 & 1363480 & 250263 & 70597 & 13539 & 136492 & 166457 & 181709 & 37464 & 184853 & 1175282 \\
\hline 28 & 3860811 & 630397 & 50389 & 1954 & 184707 & 861159 & 1155252 & 142543 & 237820 & 1617177 \\
\hline 29 & 0 & 0 & 0 & 0 & 0 & 0 & 0 & 0 & 0 & 0 \\
\hline 30 & 0 & 0 & 0 & 0 & 0 & 0 & 0 & 0 & 0 & 0 \\
\hline 31 & 375111 & 70300 & 19576 & 4883 & 36550 & 46949 & 99710 & 12355 & 52883 & 331362 \\
\hline 32 & -249536 & -140677 & 25222 & -2870 & 61449 & -832980 & 85176 & -61289 & 187478 & -1137575 \\
\hline 33 & 0 & 0 & 0 & 0 & 0 & 0 & 0 & 0 & 0 & 0 \\
\hline 34 & 0 & 0 & 0 & 0 & 0 & 0 & 0 & 0 & 0 & 0 \\
\hline 35 & 0 & 0 & 0 & 0 & 0 & 0 & 0 & 0 & 0 & 0 \\
\hline 36 & 1353463 & 639645 & 500919 & 6292791 & 1526273 & 4619844 & 257844 & 41155 & 34224 & 6941427 \\
\hline
\end{tabular}


Matriz de Contabilidad Social de Andalucía del año 2005 a precios básicos (continuación)

\begin{tabular}{|c|c|c|c|c|c|c|c|c|c|c|}
\hline & 11 & 12 & 13 & 14 & 15 & 16 & 17 & 18 & 19 & 20 \\
\hline 1 & 12376 & 57 & 15352 & 162 & 237 & 351 & 145 & 424 & 33 & 747 \\
\hline 2 & 96 & 179436 & 3027 & 47 & 757 & 275 & 61 & 211 & 93 & 2927 \\
\hline 3 & 177 & 45 & 196 & 56 & 151 & 271 & 120 & 268 & 24 & 332 \\
\hline 4 & 12 & 374 & 126282 & 2066 & 497 & 52 & 8 & 33385 & 12 & 409 \\
\hline 5 & 8 & 21 & 96703 & 965730 & 20657 & 446 & 39 & 504553 & 5177 & 9778 \\
\hline 6 & 5093 & 11069 & 290978 & 13845 & 19722 & 10379 & 2261 & 135425 & 2218 & 36006 \\
\hline 7 & 10712 & 25889 & 101860 & 54935 & 25829 & 19256 & 9914 & 112077 & 12315 & 64788 \\
\hline 8 & 2416 & 15983 & 80631 & 36855 & 17447 & 3084 & 3838 & 42068 & 3309 & 7574 \\
\hline 9 & 1354 & 1154 & 5505 & 1465 & 2363 & 2396 & 2165 & 6077 & 1033 & 4024 \\
\hline 10 & 6241 & 3815 & 5588 & 1197 & 3206 & 5722 & 2519 & 5651 & 530 & 7167 \\
\hline 11 & 662785 & 8246 & 655 & 105 & 2703 & 1947 & 961 & 2926 & 585 & 83310 \\
\hline 12 & 3278 & 590721 & 13819 & 62943 & 10551 & 9870 & 1861 & 36549 & 5015 & 610527 \\
\hline 13 & 23746 & 84557 & 1064852 & 73179 & 47194 & 31817 & 38440 & 150682 & 70215 & 574969 \\
\hline 14 & 8386 & 2718 & 3593 & 737868 & 1254033 & 308372 & 466086 & 69807 & 116483 & 263081 \\
\hline 15 & 36997 & 26654 & 6203 & 11762 & 429523 & 141300 & 43095 & 40155 & 64753 & 134348 \\
\hline 16 & 12158 & 26089 & 50605 & 128053 & 82178 & 967359 & 100523 & 119200 & 104018 & 123117 \\
\hline 17 & 1010 & 282 & 1151 & 3115 & 2909 & 40315 & 231335 & 2060 & 6543 & 8743 \\
\hline 18 & 106 & 2132 & 1660 & 12964 & 53470 & 42319 & 892 & 707824 & 5527 & 25929 \\
\hline 19 & 3 & 0 & 1 & 31 & 6683 & 25 & 0 & 60 & 405489 & 149 \\
\hline 20 & 30845 & 23363 & 70321 & 595989 & 24650 & 170818 & 21827 & 28175 & 6825 & 690611 \\
\hline 21 & 6355 & 9405 & 8855 & 13935 & 156151 & 27723 & 2595 & 125324 & 93365 & 23251 \\
\hline 22 & 39466 & 32099 & 46950 & 40713 & 38727 & 74810 & 7406 & 101819 & 34806 & 116814 \\
\hline 23 & 57389 & 79966 & 202046 & 173480 & 148966 & 104768 & 37626 & 594685 & 43640 & 237486 \\
\hline 24 & 63355 & 45650 & 96593 & 61037 & 108606 & 152089 & 33448 & 160437 & 72991 & 251539 \\
\hline 25 & 15694 & 13509 & 23661 & 9450 & 64125 & 52898 & 16204 & 76396 & 36127 & 73898 \\
\hline 26 & 45 & 252 & 313 & 85 & 90 & 205 & 15 & 803 & 79 & 5350 \\
\hline 27 & 228292 & 215789 & 249125 & 178858 & 595219 & 536310 & 172468 & 542153 & 337821 & 784405 \\
\hline 28 & 121881 & 190047 & 323740 & 250479 & 348509 & 286443 & 34547 & 712611 & 87142 & 627043 \\
\hline 29 & 0 & 0 & 0 & 0 & 0 & 0 & 0 & 0 & 0 & 0 \\
\hline 30 & 0 & 0 & 0 & 0 & 0 & 0 & 0 & 0 & 0 & 0 \\
\hline 31 & 60737 & 62484 & 67689 & 54083 & 177067 & 155795 & 56538 & 160699 & 103167 & 216337 \\
\hline 32 & 131368 & 44162 & 1556393 & 32509 & -11621 & 321872 & -36351 & -174660 & -129329 & -231271 \\
\hline 33 & 0 & 0 & 0 & 0 & 0 & 0 & 0 & 0 & 0 & 0 \\
\hline 34 & 0 & 0 & 0 & 0 & 0 & 0 & 0 & 0 & 0 & 0 \\
\hline 35 & 0 & 0 & 0 & 0 & 0 & 0 & 0 & 0 & 0 & 0 \\
\hline 36 & 2514937 & 1937061 & 5203605 & 2603582 & 861178 & 8949764 & 5590739 & 2304005 & 352083 & 2378383 \\
\hline
\end{tabular}


Matriz de Contabilidad Social de Andalucía del año 2005 a precios básicos (continuación)

\begin{tabular}{|c|c|c|c|c|c|c|c|c|c|c|}
\hline & 21 & 22 & 23 & 24 & 25 & 26 & 27 & 28 & 29 & 30 \\
\hline 1 & 1886 & 288126 & 407 & 13153 & 6771 & 15723 & 0 & 0 & 881125 & 102428 \\
\hline 2 & 459 & 113371 & 927 & 143059 & 295 & 6437 & 0 & 0 & 115630 & 31445 \\
\hline 3 & 205 & 155720 & 317 & 4071 & 291 & 4020 & 0 & 0 & 320899 & 3511 \\
\hline 4 & 7546 & 10831 & 25 & 315 & 3192 & 137 & 0 & 0 & 3817 & 4336 \\
\hline 5 & 642419 & 2741 & 547 & 749 & 16888 & 657 & 0 & 0 & 8026 & 9056 \\
\hline 6 & 701576 & 122537 & 1073607 & 82371 & 64151 & 59017 & 0 & 0 & 2047000 & 46677 \\
\hline 7 & 146800 & 589076 & 110929 & 318086 & 103060 & 196860 & 0 & 0 & 878394 & 0 \\
\hline 8 & 42524 & 134440 & 14010 & 35509 & 14650 & 16779 & 0 & 0 & 89204 & 0 \\
\hline 9 & 78085 & 158516 & 15791 & 58625 & 59945 & 50302 & 0 & 0 & 345897 & 0 \\
\hline 10 & 5674 & 3322374 & 6718 & 99258 & 6497 & 85142 & 0 & 0 & 6604500 & -620717 \\
\hline 11 & 2762 & 226936 & 1911 & 59914 & 6330 & 38863 & 0 & 0 & 2276137 & 11597 \\
\hline 12 & 584303 & 366928 & 30853 & 219176 & 24920 & 57897 & 0 & 0 & 181701 & 30056 \\
\hline 13 & 617822 & 156227 & 41038 & 1042069 & 178618 & 81488 & 0 & 0 & 1158716 & 53839 \\
\hline 14 & 436475 & 20605 & 3009 & 6885 & 7305 & 2515 & 0 & 0 & 153 & 128525 \\
\hline 15 & 1842127 & 73272 & 17846 & 37065 & 26629 & 20775 & 0 & 0 & 141168 & 426819 \\
\hline 16 & 2730627 & 599690 & 534765 & 847124 & 98792 & 190215 & 0 & 0 & 1291129 & 2380225 \\
\hline 17 & 5244 & 854505 & 171764 & 9887 & 21043 & 22884 & 0 & 0 & 2628976 & 2045208 \\
\hline 18 & 4552254 & 41114 & 6867 & 16155 & 28114 & 5338 & 0 & 0 & 61006 & 84977 \\
\hline 19 & 847 & 1186 & 46633 & 484 & 92 & 87972 & 0 & 0 & 306636 & 164347 \\
\hline 20 & 272738 & 247263 & 96594 & 498075 & 55292 & 136412 & 0 & 0 & 1683740 & 566546 \\
\hline 21 & 14469764 & 394330 & 93676 & 340328 & 1722708 & 223367 & 0 & 0 & 656707 & 27372419 \\
\hline 22 & 668603 & 2401575 & 662225 & 782894 & 198036 & 315170 & 0 & 0 & 29792850 & 1131605 \\
\hline 23 & 1528681 & 2360581 & 3175716 & 1035969 & 391567 & 298536 & 0 & 0 & 4698942 & 34709 \\
\hline 24 & 1268388 & 2310402 & 835097 & 5715961 & 1617143 & 1223199 & 0 & 0 & 9073305 & 2258430 \\
\hline 25 & 550169 & 1953377 & 391427 & 1481520 & 546726 & 377997 & 0 & 0 & 13981591 & 2234600 \\
\hline 26 & 2976 & 19237 & 7026 & 33881 & 8195 & 296354 & 0 & 0 & 433259 & 0 \\
\hline 27 & 6822328 & 8458945 & 2126278 & 13106040 & 3104337 & 6234826 & 0 & 0 & 0 & 0 \\
\hline 28 & 6767231 & 9699238 & 3754358 & 7999064 & 10167566 & 2302601 & 0 & 0 & 0 & 0 \\
\hline 29 & 0 & 0 & 0 & 0 & 0 & 0 & 47273329 & 52414709 & 0 & 0 \\
\hline 30 & 0 & 0 & 0 & 0 & 0 & 0 & 0 & 0 & 36893846 & 0 \\
\hline 31 & 2053486 & 2326028 & 626431 & 3360980 & 660742 & 1883731 & 0 & 0 & 0 & 0 \\
\hline 32 & -764726 & 992661 & 956678 & 1389075 & 3214107 & -1163140 & 0 & 0 & 0 & 0 \\
\hline 33 & 0 & 0 & 0 & 0 & 0 & 0 & 0 & 0 & 2836400 & 0 \\
\hline 34 & 0 & 0 & 0 & 0 & 0 & 0 & 0 & 0 & 7400534 & 0 \\
\hline 35 & 0 & 0 & 0 & 0 & 0 & 0 & 0 & 0 & 0 & 0 \\
\hline 36 & 0 & 0 & 3596611 & 2590236 & 369369 & 0 & 0 & 0 & 0 & 0 \\
\hline
\end{tabular}


Matriz de Contabilidad Social de Andalucía del año 2005 a precios básicos (continuación)

\begin{tabular}{|c|c|c|c|c|c|c|}
\hline & 31 & 32 & 33 & 34 & 35 & 36 \\
\hline 1 & 0 & 0 & 0 & 0 & 0 & 3482972 \\
\hline 2 & 0 & 0 & 0 & 0 & 0 & 547021 \\
\hline 3 & 0 & 0 & 0 & 0 & 0 & 211993 \\
\hline 4 & 0 & 0 & 0 & 0 & 0 & 6302 \\
\hline 5 & 0 & 0 & 0 & 0 & 0 & 82733 \\
\hline 6 & 0 & 0 & 0 & 0 & 0 & 5889326 \\
\hline 7 & 0 & 0 & 0 & 0 & 0 & 52767 \\
\hline 8 & 0 & 0 & 0 & 0 & 0 & 0 \\
\hline 9 & 0 & 0 & 0 & 0 & 11157 & 0 \\
\hline 10 & 0 & 0 & 0 & 0 & 0 & 7468804 \\
\hline 11 & 0 & 0 & 0 & 0 & 0 & 631310 \\
\hline 12 & 0 & 0 & 0 & 0 & 150 & 477929 \\
\hline 13 & 0 & 0 & 0 & 0 & 922411 & 2351884 \\
\hline 14 & 0 & 0 & 0 & 0 & 0 & 2275354 \\
\hline 15 & 0 & 0 & 0 & 0 & 0 & 637088 \\
\hline 16 & 0 & 0 & 0 & 0 & 21772 & 1420457 \\
\hline 17 & 0 & 0 & 0 & 0 & 0 & 762794 \\
\hline 18 & 0 & 0 & 0 & 0 & 0 & 647075 \\
\hline 19 & 0 & 0 & 0 & 0 & 0 & 793693 \\
\hline 20 & 0 & 0 & 0 & 0 & 30447 & 1548357 \\
\hline 21 & 0 & 0 & 0 & 0 & 0 & 0 \\
\hline 22 & 0 & 0 & 0 & 0 & 824358 & 158875 \\
\hline 23 & 0 & 0 & 0 & 0 & 84672 & 1787985 \\
\hline 24 & 0 & 0 & 0 & 0 & 13215474 & 1044126 \\
\hline 25 & 0 & 0 & 0 & 0 & 430086 & 54370 \\
\hline 26 & 0 & 0 & 0 & 0 & 12250089 & 0 \\
\hline 27 & 0 & 0 & 0 & 0 & 0 & 0 \\
\hline 28 & 0 & 0 & 0 & 0 & 0 & 0 \\
\hline 29 & 0 & 0 & 0 & 0 & 18590016 & 8513234 \\
\hline 30 & 0 & 0 & 0 & 0 & -19005897 & 20612689 \\
\hline 31 & 0 & 0 & 0 & 0 & 0 & 0 \\
\hline 32 & 0 & 0 & 0 & 0 & 0 & 0 \\
\hline 33 & 0 & 0 & 0 & 0 & 0 & 0 \\
\hline 34 & 0 & 0 & 0 & 0 & 0 & 0 \\
\hline 35 & 13075673 & 4062128 & 2836400 & 7400534 & 0 & 0 \\
\hline 36 & 0 & 0 & 0 & 0 & 0 & 0 \\
\hline
\end{tabular}

Fuente: Fuentes (2012). 